\title{
Shannon Entropy Reinterpreted
}

\author{
L. Truffet \\ IMT-Atlantque \\ Dpt. Automatique-Productique-Informatique \\ La Chantrerie, 4 rue A. Kastler, BP 20722, Nantes Cedex 3, FRANCE \\ email: laurent.truffet@imt-atlantique.fr \\ url: http://www.emn.fr/truffet
}

July 5,2019

\begin{abstract}
In this paper we remark that Shannon entropy can be expressed as a function of the self-information (i.e. the logarithm) and the inverse of the Lambert $W$ function. It means that we consider that Shannon entropy has the trace form: $-k \sum_{i} W^{-1} \circ \ln \left(p_{i}\right)$. Based on this remark we define a generalized entropy which has as a limit the Shannon entropy. In order to facilitate the reasoning this generalized entropy is obtained by a oneparameter deformation of the logarithmic function.

Introducing a new concept of independence of two systems the Shannon additivity is replaced by a non-commutative and non-associative law which limit is the usual addition. The main properties associated with the generalized entropy are established, particularly those corresponding to statistical ensembles. The Boltzmann-Gibbs statistics is recovered as a limit. The connection with thermodynamics is also studied. We also provide a guideline for systematically defining a deformed algebra which limit is the classical linear algebra. As an illustrative example we study a generalized entropy based on Tsallis self-information. We point out possible connections between deformed algebra and fuzzy logics. Finally, noticing that the new concept of independence is based on t-norm the one-parameter deformation of the logarithm is interpreted as an additive generator of t-norms.
\end{abstract}

Keywords: Deformed logarithm, deformed exponential, deformed numbers, deformed statistical properties, deformed probabilities, deformed algebra.

\section{Introduction}

This paper is mainly motivated by three facts.

1. Within several scientific communities generalizations of the Shannon entropy have been developed. Such developements are motivated by the fact 
that apparently Boltzmann-Gibbs statistics fails to explain some observed results of physical systems with e.g. long-range interaction, long-time memory, non-Markovian systems, economic systems (see e.g. [21, Section I], [19] and references therein).

2. When we want to establish an equilibrium thermal statistics which generalizes the Boltzmann-Gibbs one we optimize a generalized entropy under some constraints. For the microcanonical ensemble and internal energy most of the constraints are of the forms:

$$
\sum_{i} u_{1}\left(p_{i}\right)=Q
$$

and

$$
\sum_{i} u_{2}\left(p_{i}\right) \varepsilon_{i}=U,
$$

respectively. Where $Q \in \mathbb{R}, p_{i}$ denotes the probability of the $i$ th-microstate. $u_{1}$ and $u_{2}$ are functions of the $p_{i}$ 's. Typical choice for $u_{1}$ and $u_{2}$ is $p_{i}^{q}$ for some $q \in \mathbb{R}$ (in such case it is sometimes called effective probability [24]). Another possible choice for $u_{1}, u_{2}$ is $\frac{p_{i}^{q}}{\sum_{j} p_{j}^{q}}$ (in such case it is called escort probability [22]).

The choice of the functions $u_{1}$ and $u_{2}$ seems not to be based on a clear procedure.

3. The linear algebra is associated with the Shannon entropy. It is natural to ask which algebraic structure is associated with a generalized entropy. Once again it appears that there is no straightforward procedure which allows to associate a generalized entropy with a 'deformed algebra' (see among others [8], [13], [2]).

There exist many different ways to define a new entropy which generalizes the Shannon entropy:

- By imposing an entropy of the form (see e.g. [15]):

$$
G\left(\sum_{i} g\left(p_{i}\right)\right)
$$

for some functions $G$ and $g$.

- By imposing a trace form of the entropy, i.e.:

$$
\sum_{i} g\left(p_{i}\right)
$$

where $g$ can have the following form

$$
g\left(p_{i}\right):=p_{i}^{q} f_{\xi}\left(p_{i}\right)
$$


with $q \in \mathbb{R}, f_{\xi}(\cdot)$ playing the role of a deformed logarithm depending on the set of parameters $\xi$.

The reader is refered to e.g. [6, Table 1], 14] for more details on such entropies.

- By noticing that the Shannon entropy can be defined as the following limit:

$$
\lim _{t \rightarrow-1} \frac{d}{d t} \sum_{i} p_{i}^{-t}
$$

and to generalize this definition by replacing the classical derivatives operator, i.e. $\frac{d}{d t}$, by Jackson or fractional derivatives (see e.g. [23]).

- More recently, by imposing that for two sets of observable states $A$ and $B$ which are independent the entropy $S(A \times B)$ satisfies

$$
S(A \times B)=S(A) \hat{+} S(B):=S(A)+S(B)+\sum_{i, j} c_{i, j} S^{i}(A) S^{j}(B) .
$$

The coefficients $c_{i, j}$ are such that $\hat{+}$ is commutative, associative and has a neutral element. For more details on the subject see e.g. [18. The composition law $\hat{+}$ can be seen as a generalization of Sugeno-Tsallis composition formula (see [20, 17]): $S(A \times B)=S(A)+S(B)+(1-q) S(A) S(B)$.

In this paper we propose to define new entropy based on a reinterpretation of the Shannon entropy which is explained hereafter.

Let $n \geq 1$ and $\Omega:=\left\{\omega_{1}, \ldots, \omega_{n}\right\}$ be the set of observable states of a given system $\mathcal{S} 1$. We associate with $\Omega$ the following application:

$$
\begin{aligned}
p^{\Omega}: & \Omega \longrightarrow[0,1] \\
& \omega_{i} \longmapsto p_{i}^{\Omega} .
\end{aligned}
$$

$p_{i}^{\Omega}$ is interpreted as a certain frequency of apparition of the observable state $\omega_{i}$. If there is no ambiguity with the context $p^{\Omega}\left(\right.$ resp. $\left.p_{i}^{\Omega}\right)$ will be simply denoted $p$ (resp. $p_{i}$ ).

The Shannon entropy is defined on $\Omega$ as follows:

$$
S_{\mathrm{Sh}}^{\Omega}:=-k \sum_{i=1}^{n} p_{i} \ln \left(p_{i}\right)
$$

where $\ln (\cdot)$ denotes the Neperian logarithm.

If there is no ambiguity $S_{\mathrm{Sh}}^{\Omega}$ is simply denoted by $S_{\mathrm{Sh}}$.

We reformulate the Shannon entropy based on:

\footnotetext{
${ }^{1}$ Usually the number of states is denoted by $W$ instead of $n$ but here $W$ denotes the Lambert function.
} 
- The notion of self-information 2 , i.e. the function:

$$
\begin{aligned}
I_{\mathrm{Sh}}: & {[0,1] \longrightarrow[-\infty, 0] } \\
& p_{i} \longmapsto \ln \left(p_{i}\right) .
\end{aligned}
$$

- And $W^{-1}$ the inverse of the Lambert $\mathrm{W}$ function, i.e.:

$$
\begin{aligned}
W^{-1}: & \mathbb{R} \longrightarrow \mathbb{R} \\
& x \longmapsto x e^{x} .
\end{aligned}
$$

Shannon entropy is thus reformulated as the following trace form:

$$
S_{\mathrm{Sh}}=-k \sum_{i=1}^{n} W^{-1} \circ I_{\mathrm{Sh}}\left(p_{i}\right)=-k \sum_{i=1}^{n} I_{\mathrm{Sh}}\left(p_{i}\right) e^{I_{\mathrm{Sh}}\left(p_{i}\right)} .
$$

In the sequel the positive (Boltzmann) constant $k$ whose values depends on the particular units to be used is set for simplicity equal to 1 .

From the trace form (17) we remark that to obtain a generalized entropy it is sufficient to generalize the notion of self-information $I_{\mathrm{Sh}}$. For seek of simplicity we assume that the generalized self-information only depends upon one real parameter, say $a$, and is denoted $I_{a}$. In other words we just have to deform the logarithmic function to obtain a deformed entropy which generalizes the Shannon entropy. Thus, we introduce the following function:

$$
\begin{aligned}
S_{a}: & {[0,1]^{n} \longrightarrow \mathbb{R} } \\
& p \longmapsto-\sum_{i=1}^{n} W^{-1} \circ I_{a}\left(p_{i}\right),
\end{aligned}
$$

with:

$$
I_{a}:[0,1] \rightarrow[-\infty, 0] .
$$

We require that there exists $V \subseteq \mathbb{R}$ such that for all $a \in V$ the function $S_{a}$ satisfies the first three Shannon-Khinchin axioms. It has been shown to be equivalent to assume (see e.g. 5]) that for all $a \in V$ :

- (A1). $W^{-1} \circ I_{a}$ is convex on $[0,1]$

- (A2). $W^{-1} \circ I_{a}$ is continuous

- (A3). $W^{-1} \circ I_{a}(0)=0$

The reformulation of Shannon entropy (7) based on self-information suggests to introduce the following definitions.

The main definitions of this paper are hereafter.

- $a$-deformed number. Let $x \in[0,1]$ let us define:

$$
[x]_{a}:=e^{I_{a}(x)} .
$$

When $x$ is interpreted as a probability then we use the term $a$-probability.

\footnotetext{
${ }^{2}$ In the litterature the self-information or surprisal is defined as a nonnegative quantity which is the opposite of the one used in this paper.
} 
- $a$-deformed statistics. The $a$-expectation value $\langle O\rangle_{a}$ of an observable $O$ is defined by:

$$
\langle O\rangle_{a}:=\sum_{i}\left[p_{i}\right]_{a} O_{i}
$$

where $O_{i}$ is the value of $O$ at state $i,\left[p_{i}\right]_{a}$ is an $a$-probability. Note that $\langle 1\rangle_{a}$ corresponds to the particular case where $\forall i, O_{i}=1$.

- $a$-independence. Let us consider two sets of observable states $\Omega=\left\{\omega_{1}, \ldots, \omega_{n}\right\}$ and $\Omega^{\prime}=\left\{\omega_{1}^{\prime}, \ldots, \omega_{m}^{\prime}\right\}$ of systems $\mathcal{S}$ and $\mathcal{S}^{\prime}$, respectively. The two systems $\mathcal{S}$ and $\mathcal{S}^{\prime}$ are said to be $a$-independent if:

$$
\forall i=1, \ldots, n ; \forall j=1, \ldots, m, \quad\left[p_{i j}^{\Omega \times \Omega^{\prime}}\right]_{a}=\left[p_{i}^{\Omega}\right]_{a}\left[p_{j}^{\Omega^{\prime}}\right]_{a}
$$

where $\left[p_{i j}^{\Omega \times \Omega^{\prime}}\right]_{a},\left[p_{i}^{\Omega}\right]_{a}$ and $\left[p_{j}^{\Omega^{\prime}}\right]_{a}$ denote the $a$-probabilities of being in state $\left(\omega_{i}, \omega_{j}^{\prime}\right), \omega_{i}$ and $\omega_{j}^{\prime}$, respectively.

Remark 1.1 Note that an a-probability vector $\left(\left[p_{1}\right]_{a}, \ldots,\left[p_{n}\right]_{a}\right)$ is a nonnegative vector which is not a probability vector in general, i.e.:

$$
\sum_{i=1}^{n}\left[p_{i}\right]_{a} \neq 1
$$

And thus, an a-expectation is not the mean value associated with a random variable defined on $\Omega=\left\{\omega_{1}, \ldots, \omega_{n}\right\}$. Maybe one of the simplest possible choices for $I_{a}(x)$ is a $\ln (x)$ with $a \geq 0$. This choice leads to an a-probability vector of the well-known form: $\left(p_{1}^{a}, \ldots, p_{n}^{a}\right)$. And we refer to e.g. 24, Sections 1 and 2] who discussed the interpretation of an effective probability in the context of incomplete statistics. Since $I_{a} \leq 0$ we have $e^{I_{a}} \leq 1$ and this discussion could also be applied to the more general case of an a-probability vector.

The results and the organization of the paper are as follows. In Section 2 main results of the paper are presented. We follow the approach developed in [7]. We assume some smoothness of $I_{a}$ (i.e. once derivability) that $I_{a}$ has an inverse denoted $I_{a}^{-1}$ and that there exists $b \in \bar{V}^{3}$ such that the $\rightarrow b$ limit of $I_{a}$ is $\ln$ and the $\rightarrow b$ limit of $I_{a}^{-1}$ is the exponential function $e$. Thus, a natural choice for $u_{1}$, $u_{2}$ in equations (11) and (2) is $e^{I_{a}\left(p_{i}\right)}$ which $\rightarrow b$ limit is $p_{i}$. In subsection 2.1 we prove that $S_{a}\left(p_{1}, \ldots, p_{n}\right)$ is maximal when $\forall i=1, \ldots n: \quad p_{i}=I_{a}^{-1} \circ \ln \left(\frac{\langle 1\rangle_{a}}{n}\right)$. For two systems assumed to be $a$-independent the Shannon additivity is replaced by a non-commutative and non-associative law of the form:

$$
(x, y) \mapsto \rho x+\theta y
$$

which $\rightarrow b$ limit is the usual addition. This is the result of subsection 2.2. Properties corresponding to statistical ensembles are studied in subsection 2.3

\footnotetext{
${ }^{3} \bar{V}$ denotes the closure of the set $V$
} 
And in subsection 2.4 a connection with thermodynamics is established. Finally, in subsection 2.5 we give a way to systematically define deformed addition $\stackrel{a}{\oplus}$ by requiring that:

$$
e^{I_{a}(x \stackrel{a}{\oplus} y)}=e^{I_{a}(x)}+e^{I_{a}(y)}
$$

which leads to

$$
x \stackrel{a}{\oplus} y:=I_{a}^{-1} \circ \ln \left(e^{I_{a}(x)}+e^{I_{a}(y)}\right)
$$

and define a multiplication $\stackrel{a}{\otimes}$ by requiring that:

$$
e^{I_{a}(x \otimes y)}=e^{I_{a}(x)} \times e^{I_{a}(y)}
$$

which leads to

$$
x \stackrel{a}{\otimes} y:=I_{a}^{-1} \circ \ln \left(e^{I_{a}(x)} \times e^{I_{a}(y)}\right) .
$$

This way of defining operations seems to be known at least since [9]. Noticing that $I_{a}^{-1} \circ \ln$ is the inverse function of $e \circ I_{a}$ the deformed multiplication distributes over the deformed addition. Moreover, these operations are associative and commutative. These operations are particular cases of pseudo-addition and pseudo-multiplication which appear in the context of pseudo-analysis (see e.g. [11] and references therein).

In Section 3 we illustrate the results of Section 2, The deformed selfinformation we use is the log-exp transform of the Schweizer-Sklar-Tsallis logarithm also known as Frank generator [12].

In Section 4 we conclude by recalling the main features of the paper. We point out a possible connection between the deformed algebra developed in Section 3 and t-norm fuzzy logics. Finally, as suggested by one of the reviewers we suggest a way of inverting our approach: i.e. given a non-standard notion of independence we show that it seems possible to derive a deformed self-information and then a deformed entropy.

\section{Main results}

In this Section we follow the methodology developed in e.g. 3$]$ and 20 .

Let $V \subseteq \mathbb{R}$ and for all $a \in V$ let $S_{a}$ be the function defined by (8). Because $I_{a}$ is $\leq 0$ and the assumption (A1) $S_{a}$ is clearly a nonnegative Schur-concave function.

In the sequel we assume that $I_{a}$ is a smooth function which acts as a deformed logarithm. Thus, $I_{a}$ satisfies the following assumptions:

- (I1). $I_{a}$ is invertible on $I_{a}([0,1])$. It's inverse is denoted $I_{a}^{-1}$.

- (I2). $\exists b \in \bar{V}, \forall x \in[0,1] \lim _{a \rightarrow b} I_{a}(x)=I_{\mathrm{Sh}}(x), \quad \lim _{a \rightarrow b} I_{a}^{-1}(x)=$ $I_{\mathrm{Sh}}^{-1}(x)$.

- (I3). Smoothness: $I_{a}$ is derivable on $(0,1]$. And $I_{a}^{\prime} \neq 0$ on $(0,1)$. 


\subsection{Microcanonical ensemble}

We are looking for a candidate of the following optimization problem:

$$
\max _{p}\left\{S_{a}: \sum_{i=1}^{n}\left[p_{i}\right]_{a}=\langle 1\rangle_{a}\right\} .
$$

Where $\langle 1\rangle_{a}$ is assumed to be independent of the $p_{i}$ 's.

Let us introduce the $\lambda_{a}$ Lagrange parameter and define the function:

$$
\begin{aligned}
\phi\left(p, \lambda_{a}\right) & :=S_{a}+\lambda_{a}\left(\sum_{i=1}^{n}\left[p_{i}\right]_{a}-\langle 1\rangle_{a}\right) \\
& =-\sum_{i=1}^{n} I_{a}\left(p_{i}\right) e^{I_{a}\left(p_{i}\right)}+\lambda_{a}\left(\sum_{i=1}^{n} e^{I_{a}\left(p_{i}\right)}-\langle 1\rangle_{a}\right) .
\end{aligned}
$$

Because $I_{a}$ is derivable (see $(\mathbf{I} \mathbf{3})$ ) we have:

$$
\frac{\partial \phi}{\partial p_{i}}=I_{a}^{\prime}\left(p_{i}\right) e^{I_{a}\left(p_{i}\right)}\left(-1-I_{a}\left(p_{i}\right)+\lambda_{a}\right)
$$

and

$$
\frac{\partial \phi}{\partial \lambda_{a}}=\sum_{i=1}^{n}\left[p_{i}\right]_{a}-\langle 1\rangle_{a} .
$$

Imposing $\forall i=1, \ldots, n$ : $\frac{\partial \phi}{\partial p_{i}}=0$ and $\frac{\partial \phi}{\partial \lambda_{a}}=0$ one obtains because $I_{a}^{\prime} \neq 0$ (see (I3)):

$$
\begin{aligned}
\forall i, \quad I_{a}\left(p_{i}\right) & =-1+\lambda_{a} \\
-1+\lambda_{a} & =\ln \left(\frac{\langle 1\rangle_{a}}{n}\right) .
\end{aligned}
$$

Thus,

$$
\forall i, p_{i}=I_{a}^{-1} \circ \ln \left(\frac{\langle 1\rangle_{a}}{n}\right) .
$$

Under this condition on the $p_{i}$ 's we have

$$
S_{a}=\langle 1\rangle_{a} \ln (n)
$$

The $p_{i}$ 's correspond to a kind of deformed uniform law on $\Omega$. By assumption (I2) on $I_{a}$ the $\rightarrow b$ limit of $\langle 1\rangle_{a}$ is 1 then the $\rightarrow b$ limit of the $p_{i}$ 's corresponds to the equiprobability case. And it is immediately verified that the Boltzmann expression is recovered as the $\rightarrow b$ limit of $S_{a}$, i.e.:

$$
S_{b}=\ln (n)
$$

\subsection{Additivity}

Let us consider two sets of observable states $\Omega=\left\{\omega_{1}, \ldots, \omega_{n}\right\}$ and $\Omega^{\prime}=$ $\left\{\omega_{1}^{\prime}, \ldots, \omega_{m}^{\prime}\right\}$ of systems $\mathcal{S}$ and $\mathcal{S}^{\prime}$, respectively.

We have the following result. 
Proposition 1 If the systems $\mathcal{S}$ and $\mathcal{S}^{\prime}$ are a-independent then

$$
S_{a}^{\Omega \times \Omega^{\prime}}=\langle 1\rangle_{a}^{\prime} S_{a}^{\Omega}+\langle 1\rangle_{a} S_{a}^{\Omega^{\prime}},
$$

where $\sum_{i=1}^{n}\left[p_{i}^{\Omega}\right]_{a}=\langle 1\rangle_{a}$ and $\sum_{j=1}^{m}\left[p_{j}^{\Omega^{\prime}}\right]_{a}=\langle 1\rangle_{a}^{\prime}$.

Proof. By definition of $S_{a}^{\Omega \times \Omega^{\prime}}$ we have:

$$
S_{a}^{\Omega \times \Omega^{\prime}}=-\sum_{i=1}^{n} \sum_{j=1}^{m}\left[p_{i j}^{\Omega \times \Omega^{\prime}}\right]_{a} I_{a}\left(p_{i j}^{\Omega \times \Omega^{\prime}}\right),
$$

Now, assuming the $a$-independence of the two systems $\mathcal{S}$ and $\mathcal{S}^{\prime}$, by definition of $[\cdot]_{a}$ and $I_{a}$ we have: $I_{a}\left(p_{i j}^{\Omega \times \Omega^{\prime}}\right)=I_{a}\left(p_{i}^{\Omega}\right)+I_{a}\left(p_{j}^{\Omega^{\prime}}\right)$ and thus:

$$
S_{a}^{\Omega \times \Omega^{\prime}}=-\sum_{i=1}^{n} \sum_{j=1}^{m}\left[p_{i}^{\Omega}\right]_{a}\left[p_{j}^{\Omega^{\prime}}\right]_{a}\left(I_{a}\left(p_{i}^{\Omega}\right)+I_{a}\left(p_{j}^{\Omega^{\prime}}\right)\right)
$$

By definition of $S_{a}^{\Omega}$ and $S_{a}^{\Omega^{\prime}}$ and recalling that: $\sum_{i=1}^{n}\left[p_{i}^{\Omega}\right]_{a}=\langle 1\rangle_{a}$ and $\sum_{j=1}^{m}\left[p_{j}^{\Omega^{\prime}}\right]_{a}=$ $\langle 1\rangle_{a}^{\prime}$ the result is proved.

The composition law $(x, y) \mapsto\langle 1\rangle_{a}^{\prime} x+\langle 1\rangle_{a} y$ is neither commutative nor associative in general except in the case where $\langle 1\rangle_{a}=\langle 1\rangle_{a}^{\prime}=1$.

Taking the $\rightarrow b$ limit we retrieve the Shannon entropy additivity. The $b$ independence of the systems $\mathcal{S}$ and $\mathcal{S}^{\prime}$ reduces to the classical probabilistic independence of $\mathcal{S}$ and $\mathcal{S}^{\prime}$.

\subsection{Canonical ensemble}

We want to find a candidate to the following optimization problem:

$$
\max _{p}\left\{S_{a}: \sum_{i=1}^{n}\left[p_{i}\right]_{a}=\langle 1\rangle_{a}, \quad \sum_{i=1}^{n}\left[p_{i}\right]_{a} \varepsilon_{i}=U_{a}\right\} .
$$

To do this let us introduce the Lagrange parameters $\lambda_{a}$ and $\mu_{a}$ and define the following function:

$$
\psi\left(p, \lambda_{a}, \mu_{a}\right):=S_{a}+\lambda_{a}\left(\sum_{i=1}^{n}\left[p_{i}\right]_{a}-\langle 1\rangle_{a}\right)+\mu_{a}\left(\sum_{i=1}^{n}\left[p_{i}\right]_{a} \varepsilon_{i}-U_{a}\right) .
$$

Imposing $\forall i=1, \ldots, n: \frac{\partial \psi}{\partial p_{i}}=0, \frac{\partial \psi}{\partial \lambda_{a}}=0$ and $\frac{\partial \psi}{\partial \mu_{a}}=0$ one obtains:

$$
\begin{array}{lll}
\text { (a) } \quad \forall i, & I_{a}\left(p_{i}\right) & =-1+\lambda_{a}+\mu_{a} \varepsilon_{i} \\
\text { (b) } & e^{-1+\lambda_{a}} Z\left(\mu_{a}\right) & =\langle 1\rangle_{a} \\
\text { (c) } & e^{-1+\lambda_{a}} \frac{\partial Z}{\partial \mu_{a}} & =U_{a},
\end{array}
$$

where

$$
Z\left(\mu_{a}\right):=\sum_{i=1}^{n} e^{\mu_{a} \varepsilon_{i}}
$$


Thus, one deduces that:

$$
\forall i, I_{a}\left(p_{i}\right)=-\ln \left(Z\left(\mu_{a}\right)\right)+\ln \left(\langle 1\rangle_{a}\right)+\mu_{a} \varepsilon_{i} .
$$

Or, equivalently that:

$$
\forall i, p_{i}=I_{a}^{-1} \circ \ln \left(\frac{\langle 1\rangle_{a} e^{\mu_{a} \varepsilon_{i}}}{Z\left(\mu_{a}\right)}\right)
$$

Integrating (21, (b) and (c)) one obtains:

$$
Z\left(\mu_{a}\right)=e^{\mu_{a} \frac{U_{a}}{\langle 1\rangle_{a}}} .
$$

Thus, one derives a second expression for the $p_{i}$ 's as follows:

$$
\forall i, p_{i}\left(\varepsilon_{i}, U_{a}\right)=I_{a}^{-1} \circ \ln \left(\langle 1\rangle_{a} e^{\mu_{a}\left(\varepsilon_{i}-\frac{U_{a}}{\langle 1\rangle_{a}}\right)}\right)
$$

which is invariant by the transformation $\left(\varepsilon_{i}, U_{a}\right) \rightarrow\left(\varepsilon_{i}+c, U_{a}+\langle 1\rangle_{a} c\right)$ for $c \in \mathbb{R}$.

By assumption (I2) on $I_{a}$ it is immediately verified that in the $\rightarrow b$ limit one retrieves the Gibbs measure, i.e.:

$$
\forall i, p_{i}=\frac{e^{\mu_{b} \varepsilon_{i}}}{Z\left(\mu_{b}\right)}=e^{\mu_{b}\left(\varepsilon_{i}-U_{b}\right)} .
$$

Which is invariant by the transformation $\left(\varepsilon_{i}, U_{b}\right) \rightarrow\left(\varepsilon_{i}+c, U_{a}+c\right)$.

\subsection{Generalized temperature and free energy}

From the expression (23) we derive the generalized temperature as follows.

$$
\begin{aligned}
S_{a} & =-\sum_{i=1}^{n}\left(-\ln \left(Z\left(\mu_{a}\right)\right)+\ln \left(\langle 1\rangle_{a}\right)+\mu_{a} \varepsilon_{i}\right) e^{\left.-\ln \left(Z\left(\mu_{a}\right)\right)+\ln \left(\langle 1\rangle_{a}\right)+\mu_{a} \varepsilon_{i}\right)} \\
& =-\sum_{i=1}^{n}\left(-\ln \left(Z\left(\mu_{a}\right)\right)+\ln \left(\langle 1\rangle_{a}\right)\right) e^{\left.-\ln \left(Z\left(\mu_{a}\right)\right)+\ln \left(\langle 1\rangle_{a}\right)+\mu_{a} \varepsilon_{i}\right)} \\
& -\sum_{i=1}^{n} \mu_{a} \varepsilon_{i} e^{\left.-\ln \left(Z\left(\mu_{a}\right)\right)+\ln \left(\langle 1\rangle_{a}\right)+\mu_{a} \varepsilon_{i}\right)}
\end{aligned}
$$

Based on (21), (22) and (23) we have:

$$
-\sum_{i=1}^{n}\left(-\ln \left(Z\left(\mu_{a}\right)\right)+\ln \left(\langle 1\rangle_{a}\right)\right) e^{\left.-\ln \left(Z\left(\mu_{a}\right)\right)+\ln \left(\langle 1\rangle_{a}\right)+\mu_{a} \varepsilon_{i}\right)}=\langle 1\rangle_{a}\left(\ln \left(Z\left(\mu_{a}\right)\right)-\ln \left(\langle 1\rangle_{a}\right)\right),
$$

and

$$
\sum_{i=1}^{n} \mu_{a} \varepsilon_{i} e^{\left.-\ln \left(Z\left(\mu_{a}\right)\right)+\ln \left(\langle 1\rangle_{a}\right)+\mu_{a} \varepsilon_{i}\right)}=\mu_{a} U_{a} .
$$

Thus,

$$
S_{a}=\langle 1\rangle_{a}\left(\ln \left(Z\left(\mu_{a}\right)\right)-\ln \left(\langle 1\rangle_{a}\right)\right)-\mu_{a} U_{a} .
$$

So, defining $\frac{1}{T_{a}}:=\frac{\partial S_{a}}{\partial U_{a}}$ we have: 


$$
\frac{1}{T_{a}}=-\mu_{a}
$$

Defining the free energy as $F_{a}:=U_{a}-T_{a} S_{a}$, one obtains that:

$$
F_{a}=\frac{\langle 1\rangle_{a}}{\mu_{a}}\left(\ln \left(Z\left(\mu_{a}\right)\right)-\ln \left(\langle 1\rangle_{a}\right),\right.
$$

which recovers for $a \rightarrow b$ the classical limit, namely $F_{b}=\frac{1}{\mu_{b}} \ln \left(Z\left(\mu_{b}\right)\right)$.

\subsection{A guideline to obtaining deformed algebra and calcu- lus}

Let us consider a function $I_{a}$ satisfying assumptions (I1)-(I3). Moreover, for seek of simplicity let us assume that $I_{a}(0)=-\infty$ and $I_{a}(1)=0$.

Let us recall that deformed addition (see (12)) and multiplication (see (13)) are defined as follows:

$$
x \stackrel{a}{\oplus} y:=I_{a}^{-1} \circ \ln \left(e^{I_{a}(x)}+e^{I_{a}(y)}\right)
$$

and

$$
x \stackrel{a}{\otimes} y:=I_{a}^{-1} \circ \ln \left(e^{I_{a}(x)} \times e^{I_{a}(y)}\right)=I_{a}^{-1}\left(I_{a}(x)+I_{a}(y)\right) .
$$

By their structure these operations are commutative and associative. 0 (resp. 1) is the neutral element for $\stackrel{a}{\oplus}$ (resp. $\stackrel{a}{\otimes}$ ) and $\stackrel{a}{\otimes}$ distributes over $\stackrel{a}{\oplus}$.

By assumptions on $I_{a}$ it is clear that the $\rightarrow b$ limit of $\stackrel{a}{\oplus}$ and $\stackrel{a}{\otimes}$ are the usual addition and multiplication, respectively.

Taking care about the use of logarithmic function it is possible to define a deformed substraction and a deformed division as follows:

$$
x \stackrel{a}{\ominus} y:=I_{a}^{-1} \circ \ln \left(e^{I_{a}(x)}-e^{I_{a}(y)}\right)
$$

and

$$
x \stackrel{a}{\oslash} y:=I_{a}^{-1} \circ \ln \left(\frac{e^{I_{a}(x)}}{e^{I_{a}(y)}}\right) .
$$

Their $\rightarrow b$ limits correspond to the usual substraction and division.

For a small element $d x$ one can define:

$$
(x+d x) \stackrel{a}{\ominus} x=I_{a}^{-1} \circ \ln \left(I_{a}^{\prime}(x) e^{I_{a}(x)} d x\right)
$$

and

$$
f(x+d x) \stackrel{a}{\ominus} f(x)=I_{a}^{-1} \circ \ln \left(I_{a}^{\prime}(f(x)) e^{I_{a}(f(x))} f^{\prime}(x) d x\right) .
$$

This leads to propose as deformed derivative the following limit:

$$
\begin{aligned}
D_{a} f(x) & :=\lim _{d x \rightarrow 0}(f(x+d x) \stackrel{a}{\ominus} f(x)) \stackrel{a}{\ominus}((x+d x) \stackrel{a}{\ominus} x) \\
& =I_{a}^{-1} \circ \ln \left(\frac{\left(I_{a}^{\prime}(f(x)) e^{I_{a}(f(x))}\right.}{I_{a}^{\prime}(x) e^{I_{a}(x)}} f^{\prime}(x)\right)
\end{aligned}
$$


If

(I4): $\lim _{a \rightarrow b} I_{a}^{\prime}(x)=\frac{1}{x}$

then the $\rightarrow b$ limit of $D_{a} f$ is $f^{\prime}$.

By noticing that:

$$
\begin{gathered}
e^{I_{a}\left(D_{a} f(x)\right)}=\frac{I_{a}^{\prime}(f(x)) e^{I_{a}(f(x))}}{I_{a}^{\prime}(x) e^{I_{a}(x)}} f^{\prime}(x), \\
D_{a} f(x) \stackrel{a}{\otimes} g(x)=I_{a}^{-1} \circ \ln \left(e^{I_{a}\left(D_{a} f(x)\right)} \times e^{I_{a}(g(x))}\right) \\
D_{a} f(x) \stackrel{a}{\otimes} g(x) \stackrel{a}{\oplus} f(x) \stackrel{a}{\otimes} D_{a} g(x)=I_{a}^{-1} \circ \ln \left(\frac{\left(I_{a}(f(x))+I_{a}(g(x))\right)^{\prime}}{I_{a}^{\prime}(x) e^{I_{a}(x)}} e^{I_{a}(f(x))+I_{a}(g(x))}\right)
\end{gathered}
$$

and that:

$$
\begin{aligned}
D_{a}(f(x) \stackrel{a}{\otimes} g(x)) & =I_{a}^{-1} \circ \ln \left(\frac{I_{a}^{\prime}(f(x) \otimes g(x)) e^{I_{a}(f(x) \stackrel{a}{\otimes} g(x))}}{I_{a}^{\prime}(x) e^{I_{a}(x)}}(f(x) \stackrel{a}{\otimes} g(x))^{\prime}\right) \\
& =I_{a}^{-1} \circ \ln \left(\frac{\left(I_{a}(f(x) \stackrel{a}{\otimes}(x))\right)^{\prime}}{I_{a}^{\prime}(x) e^{I_{a}(x)}} e^{I_{a}(f(x)+g(x))}\right) \\
& =I_{a}^{-1} \circ \ln \left(\frac{\left(I_{a}(f(x))+I_{a}(g(x))\right)^{\prime}}{I_{a}^{\prime}(x) e^{I_{a}(x)}} e^{I_{a}(f(x)+g(x))}\right)
\end{aligned}
$$

the Leibniz rule is verified, i.e.:

$$
D_{a}(f(x) \stackrel{a}{\otimes} g(x))=D_{a} f(x) \stackrel{a}{\otimes} g(x) \stackrel{a}{\oplus} f(x) \stackrel{a}{\otimes} D_{a} g(x) .
$$

We define a deformed integral $\int^{a}$ by requiring that $D_{a} \int^{a} f=f$ and we obtain:

$$
\int^{a} f=I_{a}^{-1} \circ \ln \left(\int I_{a}^{\prime}(x) e^{I_{a}(x)} e^{I_{a}(f(x))} d x\right)
$$

where $\int$ denotes the Lebesgue integral and it is assumed that $x \mapsto I_{a}^{\prime}(x) e^{I_{a}(x)} e^{I_{a}(f(x))}$ is Lebesgue integrable.

We also remark that under the assumptions (I1)-(I4) and if (I5): $\exists h \geq 0$ Lebesgue integrable s.t.:

$$
\forall a, \forall x:\left|I_{a}^{\prime}(x) e^{I_{a}(x)} e^{I_{a}(f(x))}\right| \leq h(x)
$$

the $\rightarrow b$ limit of $\int^{a}$ is $\int$ (see e.g.[16, p. 94]).

From definition (33) one easily verifies that:

$$
\int^{a} f \stackrel{a}{\oplus} g=\int^{a} f \stackrel{a}{\oplus} \int^{a} g
$$




\section{An illustrative example: generalized entropy based on Schweizer-Sklar-Tsallis self-information}

As an example, let $a \in \mathbb{R}_{+} \backslash\{0\}$. And let the Tsallis self-information defined by (see e.g. [20]):

$$
\forall x \in[0,1], I_{a}^{\mathrm{\top s}}(x):=\frac{x^{a}-1}{a} .
$$

From Tsallis self-information let us define the following function:

$$
\forall x \in[0,1], I_{a}^{\mathrm{GTs}}(x):=\ln \circ I_{a}^{\mathrm{Ts}} \circ e^{x}-\ln \circ I_{a}^{\mathrm{Ts}} \circ e^{1}
$$

which could be rewritten as follows:

$$
\forall x \in[0,1], I_{a}^{\mathrm{GTs}}(x)=\ln \left(\frac{e^{a x}-1}{e^{a}-1}\right) .
$$

This function is also known as the Frank generator.

Let $V:=\left(0, \ln \left(1+e^{3}\right)\right]$, thus $\bar{V}=\left[0, \ln \left(1+e^{3}\right)\right]$. For all $a \in V$, it is clear that $W^{-1} \circ I_{a}^{\mathrm{GTs}}$ is continuous and Assumption (A2) is verified. Adopting the convention that $\ln (0)=-\infty$, Assumption (A3) is also verified. The convexity of $W^{-1} \circ I_{a}^{\mathrm{GTs}}$ (i.e. Assumption $(\mathbf{A} \mathbf{1})$ ) is proved in subsection 3.1 .

We remark that $I_{a}^{\mathrm{GTs}}$ is invertible and its inverse is:

$$
I_{a}^{-\mathrm{GTs}}(x)=\frac{1}{a} \ln \left(1+\left(e^{a}-1\right) e^{x}\right) .
$$

Thus, (I1) is verified.

We note that for $b=0(\mathbf{I 2})$ is verified. The derivative of $I_{a}^{\mathrm{GTs}}$ is

$$
\left(I_{a}^{\mathrm{GTs}}\right)^{\prime}(x)=\frac{a e^{a x}}{e^{a x}-1}
$$

which is defined on $(0,1]$ and $\neq 0$. Thus, $(\mathbf{I} 3)$ is verified.

Finally, we easily see that:

$$
\lim _{a \rightarrow 0}\left(I_{a}^{\mathrm{GTs}}\right)^{\prime}(x)=\frac{1}{x}
$$

thus (I4) is verified.

\subsection{Schur-concavity}

Let us denote $h:[0,1] \rightarrow[0, \infty), x \mapsto \frac{e^{a x}-1}{e^{a}-1}$. Then, we have:

$$
\frac{h(x)}{e^{a x}}\left(W^{-1} \circ I_{a}^{\mathrm{GTs} s}\right)^{\prime \prime}(x)=\frac{a^{2}}{e^{a}-1} u(x),
$$

where $(\cdot)^{\prime \prime}$ denotes the second derivative and

$$
u(x)=h(x) \ln (h(x))+2 h(x)+\frac{1}{e^{a}-1} .
$$


The derivative of $u$ is then:

$$
u^{\prime}(x)=h^{\prime}(x)(\ln (h(x))+3) .
$$

Noticing that $h^{\prime}>0$ on $[0,1]$ the function $u$ is such that $u(0)=\frac{1}{e^{a}-1}>0$, $u(1)=2+\frac{1}{e^{a}-1}>0$ and admits a minimum at $x^{*}=\ln \left(1+\left(e^{a}-1\right) e^{-3}\right)^{\frac{1}{a}}$ which is $u\left(x^{*}\right)=-e^{-3}+\frac{1}{e^{a}-1}$. Thus, $\left(W^{-1} \circ I_{a}^{\mathrm{GTs}}\right)^{\prime \prime} \geq 0$ if and only if $u\left(x^{*}\right) \geq 0$ that is:

$$
a \leq \ln \left(1+e^{3}\right) .
$$

\subsection{Microcanonical ensemble}

Applying formula (17) with (37) we have:

$$
\forall i, p_{i}=\frac{1}{a} \ln \left(1+\left(e^{a}-1\right) \frac{\langle 1\rangle_{a}}{n}\right) .
$$

And by (18) we have:

$$
S_{a}=\langle 1\rangle_{a} \ln (n) .
$$

Note that the $\rightarrow 0$ limit of $p_{i}$ is $\frac{1}{n}$.

\subsection{Canonical ensemble}

Applying formula (24) with (37) we have:

$$
\forall i, p_{i}=\frac{1}{a} \ln \left(1+\left(e^{a}-1\right) \frac{e^{\mu_{a} \varepsilon_{i}}}{Z\left(\mu_{a}\right)}\right)
$$

\subsection{Deformed algebra}

Following subsection 2.5 and noticing that $I_{a}^{-\mathrm{GT}} \circ \ln (x)=\frac{1}{a} \ln \left(1+\left(e^{a}-1\right) x\right)$, $e^{I_{a}^{\mathrm{GT}}(x)}=\frac{e^{a x}-1}{e^{a}-1}$ we define the deformed addition:

$$
x \stackrel{a}{\oplus} y:=\frac{1}{a} \ln \left(e^{a x}+e^{a y}-1\right)
$$

the deformed multiplication which is a particular case of copula (see e.g. [1, Table 2.6]):

$$
x \stackrel{a}{\otimes} y:=\frac{1}{a} \ln \left(1+\frac{\left(e^{a x}-1\right)\left(e^{a y}-1\right)}{e^{a}-1}\right)
$$

the deformed substraction:

$$
x \stackrel{a}{\ominus} y:=\frac{1}{a} \ln \left(1+e^{a x}-e^{a y}\right)
$$

the deformed division: 


$$
x \stackrel{a}{\oslash} y:=\frac{1}{a} \ln \left(1+\left(e^{a}-1\right) \frac{e^{a x}-1}{e^{a y}-1}\right)
$$

And the deformed calculus is as follows. We define the deformed derivatives by:

$$
D_{a} f(x)=\frac{1}{a} \ln \left(1+\left(e^{a}-1\right) \frac{e^{a f(x)}}{e^{a x}} f^{\prime}(x)\right)
$$

And the deformed integral by:

$$
\int^{a} f=\frac{1}{a} \ln \left(1+\frac{a}{e^{a}-1} \int e^{a x}\left(e^{a f(x)}-1\right) d x\right)
$$

And we see that:

$$
\begin{array}{ll}
\stackrel{0}{\oplus} & =+, \\
0 & =\times, \\
\ominus^{0} & =-, \\
\oslash^{0} & =/, \\
D_{0} f & =f^{\prime}, \\
\int^{0} f & =\int f .
\end{array}
$$

\section{Conclusion}

To conclude let us recall the main features of this paper. Based on the remark that Shannon entropy can be expressed as a function of the self-information (i.e. the logarithm) and the inverse of the Lambert $W$ function a new definition of generalized entropy has been proposed which limit is the classical Shannon entropy.

Axioms that characterize one-parameter deformation of the logarithmic and exponential functions were proposed (see (I1)-(I3)). A notion of one-parameter deformed probability and independence of systems were also introduced. By using a standard variational principle microcanonical and canonical distributions have been established. The new notion of independence leads to define a non-commutative and non-associative composition law which limit is the ussual addition.

The generalized entropy proposed in this paper allows us to define a generalized temperature based on a fundamental relation of the standard thermodynamics. It was also possible to define a generalized free energy. All these quantities converge to the standard ones.

A systematic way to define a 'deformed algebra' and a 'deformed calculus' was provided. The deformed algebra and calculus converge to the standard ones. Some of the operations defined in Section 3 are knonw as triangular norms or copulas (see e.g. [10]). They appear in other contexts such as fuzzy logic and statistics. For example the multiplication defined by (39) is a triangular norm 
on $[0,1]$. Note that this multiplication is also known as Frank copula (see e.g. [12]). With 0 interpreted as falsity and 1 as truth a triangular norm is an operation which is commutative, associative, monotone, with neutral element 1 and such that $0 \stackrel{a}{\otimes} x=0$ and continuous. A t-norm is interpreted in fuzzy logic as a conjunction. The residuum of $\stackrel{a}{\otimes}$ defined by (39) which is denoted $\stackrel{a}{\Rightarrow}$ and defined as:

$$
x \stackrel{a}{\Rightarrow} y:=\sup \{z \in[0,1]: z \stackrel{a}{\otimes} x \leq y\}=\min (1, y \stackrel{a}{\oslash} x)
$$

where $\stackrel{a}{\oslash}$ is the division defined by (41). Thus, roughly speaking the division plays the role of the implication in fuzzy logic. Using the standard negation $\sim: x \mapsto 1-x$ one obtains a disjunction $\stackrel{a}{\mathrm{OR}}$ as follows:

$$
x \stackrel{a}{\text { OR }} y:=1-(1-x) \stackrel{a}{\otimes}(1-y) .
$$

Thus, interpreting $\leq$ as the deduction operator the structure $([0,1], \stackrel{a}{\otimes}, \sim, \stackrel{a}{\mathrm{O} R}, \stackrel{a}{\Rightarrow}$ $, 0,1, \leq)$ plays the same role in fuzzy logic than the Boolean algebra in classical logic.

Finally, let us make the following remarks. (A) The $a$-independence of two systems (11) is equivalent to:

$$
p_{i j}^{\Omega \times \Omega^{\prime}}=p_{i}^{\Omega} \stackrel{a}{\otimes} p_{j}^{\Omega^{\prime}}=I_{a}^{-1}\left(I_{a}\left(p_{i}^{\Omega}\right)+I_{a}\left(p_{j}^{\Omega^{\prime}}\right)\right)
$$

by definition of $[\cdot]_{a}$ (see Definition ??) and $\stackrel{a}{\otimes}$ (see [13). (B) By construction $\stackrel{a}{\otimes}$ is a t-norm. And t-norms are also used to express the independence of events in the context of fuzzyness [4, Definition 2.1]. Thus, starting from an a-priori given notion of independence modelled by a t-norm $T$ it is possible to derive a deformed logarithm $I_{a}$ by solving $x \stackrel{a}{\otimes} y=T(x, y)$ which is equivalent to solve the functional equation:

$$
I_{a}^{-1}\left(I_{a}(x)+I_{a}(y)\right)=T(x, y) .
$$

Thus, the deformed logarithm $I_{a}$ is interpreted as an additive generator of the t-norm $T$. As a basic example let us define $T(x, y)=x y$. Then, (44) becomes:

$$
I_{a}(x)+I_{a}(y)=I_{a}(x y)
$$

which solution is $I_{a}(x):=a \ln (x)$ with $a \geq 0$. This leads to the deformed addition being defined by: $x \stackrel{a}{\oplus} y:=\left(x^{a}+y^{a}\right)^{\frac{1}{a}}$ and the deformed entropy defined by:

$$
S_{a}:=-\sum_{i} a \ln \left(p_{i}\right) p_{i}^{a} .
$$

Now, looking back the interpration of an $a$-probability vector in the context of incomplete statistics (see Remark 1.1] in the introduction) the last point of this conclusion suggests another point of view in term of fuzzyness vs randomness for deformed statistics and thermodynamics. 


\section{Acknowledgement}

Author would like to thank referees for their comments, suggestions and remarks.

\section{References}

[1] C. Alsina, M.J Frank, and B. Schweizer. Associative Functions: Triangular Norms and Copulas. World Scientific Publishing, 2006.

[2] E. P. Borges. A Possible Deformed Algebra and Calculus Inspired in Nonextensive Thermostatistics. Physica A, 340, 2004. (95-101).

[3] E. M. F. Curado and C. Tsallis. Generalized Statistical Mechanics: Connection with Thermodynamics. J. Phys. A: Math. Gen., 24, 1991. (69-72).

[4] G. De Cooman. Possibility Theory III: Possibilistic Independence. Int. Journ. of General Systems, 25(4), 1997. (353-371).

[5] R. Hanel and S. Thurner. A Comprehensive Classification of Complex Statistical Systems and an Ab-initio Derivation of Their Entropy and Distribution Function. Europhys. Lett., 93(2), 2011. 20006.

[6] V. M. Ilic and M. S. Stankovic. A Unified Characterization of Generalized Information and Certainty Measures. Physica A, 415, 2014. (229-239).

[7] E. T. Jayne. Information Theory and Statistical Mechanics. Phys. Rev., 106(4), 1957. (620-630).

[8] N. Kalogeropoulos. Algebra and Calculus for Tsallis Thermostatistics. Physica A, 356(2-4), 2005. (408-418).

[9] G. Kaniadakis. Statistical Mechanics in the Context of Special Relativity. Phys. Rev. E, 66, 2002. 056125.

[10] E. P. Klement, Mesiar, and E. Pap. Triangular Norms. Kluwer Academics Publishers, 2000.

[11] R Mesiar and E. Pap. Idempotent Integral as Limit of g-integrals. Fuzzy Sets and Systems, 102, 1999. (385-392).

[12] R. B. Nelsen. An Introduction to Copulas. Springer, 2006. 2nd Edition.

[13] L. Nivanen, A. Le Méhauté, and Q. A. Wang. Generalized Algebra Within a Nonextensive Statistics. Rep. Math. Phys., 52(3), 2003. (437-444).

[14] Th. Oikonomou and G. Baris Bagci. A Note on The Definition of Deformed Exponential and Logarithmic Functions. Journ. Math. Phys., 50, 2009. (1033011-1033019). 
[15] A. Renyi. On Measures of Entropy and Information. In Proceedings of the 4th Berkeley Symposium on Mathematics, Statistics and Probability, vol 1, Univ. California Press, Berkeley, 1961. (547-561).

[16] H.L. Royden. Real Analysis (3rd Edition). Collier Macmillan, 1988.

[17] M. Sugeno. Theory of Fuzzy Integrals and Its Applications . PhD thesis, Tokyo Institute of Technology, Japan, 1974.

[18] P. Tempesta. Formal Groups and Z-entropies. 2016. Proc. Royal Society A.

[19] M. Tribeche and K. Ourabah. Etat de l'Art Sur la Généralisation de la Statistique de Boltzmann-Gibbs. Rev. Alg. de Physique, 2(2), 2015. (110122).

[20] C. Tsallis. Possible Generalization of Boltzmann-Gibbs Statistics. Journ. Stat. Phys., 52(1/2), 1988. (479-487).

[21] C. Tsallis. Some Comments on Boltzmann-Gibbs Statistical Mechanics. Chaos, Solitons \& Fractals, 6, 1995. (539-559).

[22] C. Tsallis, R. S. Mendes, and A. R. Plastino. The Role of The Constraints Within Generalized Nonextensive Statistics. Physica A, 261, 1998. (534$554)$.

[23] M. R. Ubriaco. Entropies Based on Fractional Calculus. Phys. Letters A, 373, 2009. (2516-2519).

[24] Q. A. Wang. Incomplete Statistics and Nonextensive Generalizations of Statistical Mechanics. Chaos, Solitons and Fractals, 12, 2001. (1431-1437). 